\title{
Antitumor activity of Cetuximab in combination with Ixabepilone on triple negative breast cancer stem cells
}

\author{
Tomonori Tanei ${ }^{1 \dagger}$, Dong Soon Choi ${ }^{1 \dagger}$, Angel A. Rodriguez ${ }^{1}$, Diana Hwang Liang ${ }^{2}$, Lacey Dobrolecki ${ }^{1}$, \\ Madhumita Ghosh ${ }^{1}$, Melissa D. Landis ${ }^{1}$ and Jenny C. Chang ${ }^{1 *}$
}

\begin{abstract}
Background: Developing novel strategies against treatment-resistant triple negative breast cancer (TNBC) cells remains a significant challenge. The ErbB family, including epidermal growth factor receptor (EGFR), plays key roles in metastasis, tumorigenesis, cell proliferation, and drug resistance. Recently, these characteristics have been linked to a small subpopulation of cells classified as cancer stem cells (CSC) which are believed to be responsible for tumor initiation and maintenance. Ixabepilone is a new generation microtubule-stabilizing agent, which has been expected to be more efficacious than conventional taxanes. Here we aim to investigate whether the EGFR monoclonal antibody Cetuximab, in combination with Ixabepilone, is more effective in eliminating CSC populations compared to chemotherapy alone in TNBC.
\end{abstract}

Methods: Representative TNBC cell lines (MDA-MB-231 and SUM159) were used to evaluate breast CSC populations. We used fluorescence-activated cell sorter analysis $\left(\mathrm{CD}_{4} 4^{+}\right.$and $\mathrm{CD} 24^{- \text {/low }}$, or Aldefluor $\left.{ }^{+}\right)$and a selfrenewal assay called mammosphere formation efficiency (MSFE) to measure CSC population size after treatment with Cetuximab, or Cetuximab plus Ixabepilone in vitro.

Results: Although there was no significant decrease in cell viability, Cetuximab reduced MSFE and the CSC population in breast cancer cells in vitro and in vivo through inhibition of autophagy. Also, SUM159 and MDA-MB231 orthotopic tumors demonstrated partial response to Centuximab or Ixabepilone monotherapy; however, the effect of the combination treatment was significant only in SUM159 tumors ( $p<0.0001)$, when compared to Ixabepilone alone.

Conclusions: Overall, our findings demonstrate that EGFR-targeted therapy by Cetuximab effectively reduces the CSC population in TNBC tumors. However, combination therapy with Ixabepilone may be effective only in a small subset of TNBCs, warranting further investigation of alternative approaches to target multiple pathways for TNBC treatment.

\section{Background}

Triple-negative breast cancer (TNBC), which accounts for $20 \%$ of all breast cancers, is characterized by the absence of estrogen receptor (ER), progesterone receptor (PR), and human epidermal growth factor receptor 2 (HER2) expression. They are histologically high grade, aggressive, and lethal tumor types that lack targeted therapeutic options. Patients with TNBC are associated

\footnotetext{
* Correspondence: jcchang@houstonmethodist.org

${ }^{\dagger}$ Equal contributors

'Methodist Cancer Center, Houston Methodist Hospital, 6445 Main Street,

P21-34, Houston, TX 77030, USA

Full list of author information is available at the end of the article
}

with relatively poor prognosis and are at a significant risk of relapse and frequent metastases [1, 2]. Triplenegative and basal-like breast cancers display a similar profile of cell-surface markers of breast cancer stem cells (CSCs) [3]. CSCs are defined as rare tumor cells that are capable of self-renewal and give rise to multipotent progenitor cells, which ultimately differentiate into all cell types within the tumor [4-6]. CSCs have been identified by cell sorting technologies using various surface markers in acute myeloid leukemia and solid tumors, including breast tumors [7]. Studying tumorigenic cells separated in vitro, from malignant human breast cancer-derived pleural effusions, Al Hajj and 
colleagues isolated a cell population characterized by high CD44 expression and low or undetectable levels of CD24 (CD $\left.44^{+} / \mathrm{CD} 24^{-/ \text {low }}\right)$. These cells had classic features of bona fide stem cells, including the capacity for selfrenewal and generation of heterogeneous progeny [8]. This subpopulation can form mammospheres in vitro and were shown to be enriched for tumorigenic cells by their ability to form xenograft tumors in immunocompromised mice [8]. Ginestier et al. demonstrated that aldehyde dehydrogenase 1 is an alternative marker for breast CSCs [8]. We have recently shown that CD $44^{+} / \mathrm{CD} 24^{-/ \text {low }}$ and $\mathrm{ALDH}^{+}$phenotypes reflect different epithelialmesenchymal transition states in CSCs [9]. Identification of breast CSCs from tumor samples or breast cancer cell lines has been based mainly on $\mathrm{CD} 44^{+} / \mathrm{CD} 24^{-/ \text {low }}$ or $\mathrm{ALDH}^{+}$phenotypes. We have previously reported that breast CSCs are a subpopulation of cells within the primary tumor responsible for tumor initiation and metastases, and are associated with resistance to chemotherapy in human breast cancers following neoadjuvant chemotherapy [10]. In addition, it has been shown that epidermal growth factor receptor (EGFR) signaling may be required for cancer self-renewal [11]. EGFR is more commonly overexpressed in TNBC than in other breast cancer subtypes $[12,13]$. Also, TNBC can be classified as basal type cancer defined by EGFR and cytokeratin 5/6 staining.

Ixabepilone is a new-generation microtubule-stabilizing agent and has more efficacious anti-tumor effects than taxanes $[14,15]$. It is an analog of epothilone B, a naturally occurring microtubule stabilizer with very high cytotoxic activities against a wide range of tumor types, including drug-resistant tumors. For example, anthracycline- and taxane-resistant metastatic breast cancers (MBCs) are known to be highly sensitive to Ixabepilone as a single agent or in combination with Capecitabine [13]. Importantly, significant anticancer activity was seen in ER, PR, HER2 negative TNBC patients with MBC. This is consistent with the preclinical activity of Ixabepilone against human cancer cell lines resistant to taxanes and other agents [16].

Combination therapy is a mainstay of anticancer treatment, with optimal combinations producing synergistic antitumor responses. This is achieved by combining agents with established safety profiles and nonoverlapping mechanisms of action. Thus, this study seeks to evaluate the combination therapy of combining Cetuximab and Ixabepilone, which might be more effective at targeting cancer stem cells than other antitubulins, as a possible way to increase antitumor activity. In the present study, we investigated whether the breast CSC population enriched for tumorigenic $\mathrm{CD} 44^{+} \mathrm{CD} 24^{-/ \text {low }}$ cells could be eradicated when treated with Cetuximab and Ixabepilone, as opposed to chemotherapy alone, in TNBC xenografts. Our findings suggest that Ixabepilone produces therapeutic synergism with Cetuximab only in a small subset of TNBCs and provides additional evidence that clinical trials using Cetuximab in combination with Ixabepilone should be applied with caution.

\section{Methods}

Cell proliferation and viability measurements (WST- 1 assay) TNBC cell lines MDA-MB-231 and SUM159 were purchased from American Type Culture Collection (ATCC), Manassas, VA, USA, and from Asterand, Detroit, MI, USA, These cell lines were chosen based on their high expression of epithelial-mesenchymal transition markers (EMT), metastatic properties, and percentage of CD44+/CD24cells. Both cell lines were grown in DMEM (Invitrogen, Carlsbad, CA) with $10 \%$ fetal bovine serum (Cellgro, Manassas, VA) and $1 \%$ penicillin/streptomycin. WST-1 assay was performed using Premixed WST-1 Cell Proliferation Reagent (Clontech, Mountain View, CA) according to the manufacturer's protocol to access cell viability. Cells were seeded into 96-well tissue culture plates at the concentration of 2,000 cells/well and incubated at different concentrations of Cetuximab (Erbitux, Bristol Myers Squibb, NJ) $(0.001-100 \mu \mathrm{g} / \mathrm{ml})$, Cetuximab $(1 \mathrm{nM}-100 \mathrm{uM})$ or in combination with Ixabepilone (Ixampra, Bristol Myers Squibb, NJ) for $72 \mathrm{~h}$. Cell viability was analyzed indirectly by measuring formazan formation by mitochondrial dehydrogenases in viable cells. The absorbance was measured using a multiwall scanning spectrophotometer (Infinite M200 pro, Tecan, Switzerland) at $440 \mathrm{~nm}$ (measurement wavelength) and $600 \mathrm{~nm}$ (reference wavelength).

\section{Fluorescence-activated cell sorting (FACS) for $\mathrm{CD} 44^{+} \mathrm{CD} 24^{-/ \text {low }}$ cell isolation}

To analyze CD $44^{+} / \mathrm{CD} 24^{-/ \text {low }}$ cells, approximately $10^{6}$ cells were resuspended in Hank's balanced salt solution (HBSS) supplemented with $2 \%$ fetal bovine serum, mixed, and incubated for 15 minutes at $4{ }^{\circ} \mathrm{C}$ with mouse monoclonal anti-CD44-APC $(0.6 \mu \mathrm{g} / 100 \mu \mathrm{l} /$ test $)$ and anti-CD24-PE (4 $\mu \mathrm{g} / 100 \mu \mathrm{l} /$ test) (BD Pharmingen, San Jose, CA, USA) according to the manufacturer's instructions. The cells were then washed and resuspended in HBSS (Invitrogen, Carlsbad, CA, USA) supplemented with 1 M HEPES. Propidium iodide (PI) was added to exclude dead cells before FACS analysis using BD LSRII flow cytometry (BD Bioscience, San Jose, CA, USA). Only live and single cell populations were gated out for population analysis using a PI gate and side and forward scatter gates. Negative and positive controls were stained with antibodies of isotype control, PE (phycoerythrin) positive control, APC (allophycocyanin) positive control, or fluorescein isothiocyanate (FITC) positive control. All experiments were repeated three times.

\section{Aldefluor (ALDF) assay}

The Aldefluor assay was performed as described by the manufacturer (Stem cell technology, BC, Canada). Briefly, 
$5 \times 10^{5}$ cells were incubated for 45 minutes at $37^{\circ} \mathrm{C}$ with ALDF cocktail (ALDF reagent; $2.5 \mu$, ALDF Buffer; $500 \mu \mathrm{l} /$ test). After washing the ALDF buffer, the cells were rinsed and resuspended in HBSS +1 M HEPES. For xenograft tumors, cells were additionally stained with anti-H2Kd-PE $(2.5 \mu \mathrm{l} / 100 \mu \mathrm{l} /$ test $)$ antibodies on ice for 15 minutes to exclude mouse components. We used cells stained with DEAB cocktail (DEAB reagent; $5 \mu \mathrm{l}$ ALDF reagent; $2.5 \mu \mathrm{l}$ ALDF buffer; $500 \mu \mathrm{l} /$ test) as negative controls.

\section{Mammosphere-forming assay}

Mammosphere-forming efficiency (MSFE) was measured by counting spheres as previously described $[17,18]$. In brief, cancer cells $(5,000$ cells $/ \mathrm{ml})$ were cultured for $72 \mathrm{~h}$ in ultralow attachment 24-well plates (Corning Costar Co., MA, USA) with the mammosphere culture medium Plus (MEGM+, Lonza, Switzerland) containing $20 \mathrm{ng} / \mathrm{ml}$ of bFGF (Invitrogen, Carlsbad, CA, USA), $20 \mathrm{ng} / \mathrm{ml}$ of EGF (Invitrogen), $4 \mu \mathrm{g} / \mathrm{ml}$ of heparin (StemCell Technologies, BC, Canada), and B27 supplement (Invitrogen). Cells were treated at the time of cell seeding with Cetuximab, Ixabepilone, or the combination at the indicated concentrations. Mammospheres were counted using the Gel Counter (Optronix, UK) and GelCount software. To calculate the MSFE, the following formula was used:

$$
\begin{aligned}
\operatorname{MSFE}(\%)= & (\text { Total number of spheres } \times 100) \\
& \div \text { Total number cells seeded }
\end{aligned}
$$

Each experiment was repeated at least three times with six replicates for each treatment group.

\section{Animal experiments}

All animal protocols were reviewed and approved by the Animal Protocol Review Committee at Houston Methodist Research Institute (Houston, TX, USA). MDA-MB-231 or SUM159 cells $\left(2 \times 10^{6}\right.$ cells $\left./ 100 \mathrm{ml}\right)$ were injected orthotopically in female immunocompromised severe combined immunodeficiency (SCID)/ Beige mice (Charles River Laboratories, Wilmington, MA, USA). After tumors reached $5-8 \mathrm{~mm}$ in diameter, mice were randomly grouped based on tumor sizes and injected intravenously with (A) control, intravenous (i.v.) PBS, (B) Cetuximab, i.v. (4 mg/kg/weekly) (C) Ixabepilone, intraperitoneal (i.p.) (10 mg/kg/weekly), (D) Cetuximab, i.v. + Ixabepilone, i.p. (equivalent dose/weekly). Tumor diameters and body weights were recorded twice per week, and mice were euthanized at the end of treatment or when the recurrent tumor size reached the basal level. The tumors were then excised, minced, and digested in mammary epithelial growth medium (MEGM) with 200$250 \mathrm{U} / \mathrm{ml}$ of Collagenase type III (Worthington, NJ) and 0.8 units/ml of Dispase (Worthington, NJ) in a shaking incubator at $37{ }^{\circ} \mathrm{C}$ for $1.5 \mathrm{~h}$. The samples were filtered, subjected to hypotonic shock ( $9 \mathrm{ml}$ of sterile $\mathrm{H}_{2} \mathrm{O}$ for 10 seconds followed with $1 \mathrm{ml}$ of $10 \times \mathrm{HBSS}$ ) to lyse red blood cells, washed with HBSS, and finally re-filtered for single cells using $40-\mu \mathrm{m}$ pore filters. To analyze MSFE, cells $(20,000$ cells $/ \mathrm{ml})$ were plated in the ultralow attachment, as described above, for 2 weeks.

\section{Western blot assay}

Western blotting was performed as previously described [17]. Briefly, cells were lysed with 1X Cell Lysis Buffer (Cell Signaling Tech., Danvers, MA, USA). After measuring the protein concentration using a Pierce BCA protein assay kit (Thermo Fisher Scientific Inc, Waltham, MA, USA), a total of $300 \mu \mathrm{g}$ of protein extract from each sample was mixed with $4 \times$ sample buffer (Bio-Rad, Hercules, CA, USA) containing $5 \% \beta$-mercaptoethanol (Sigma, St. Louis, MO, USA). Samples (30 $\mu \mathrm{g} /$ well) were loaded onto a 4-20\% SDS-PAGE gel and transferred to nitrocellulose membranes (Bio-Rad). All antibodies were purchased from Cell Signaling Technology: phosphoEGF receptor (Tyr1068) antibody, EGF receptor antibody (1 F4), phospho-AKT (Ser473) antibody, AKT antibody, phospho-extracellular signal-regulated kinase (ERK)1/2 antibody, ERK1/2 antibody, p62, LC3b, and $\beta$-Actin.

\section{Statistical analyses}

All quantitative parameters are presented as mean with standard deviation, and all results are expressed as mean \pm standard error of the mean (SEM) for in vivo experiments. In vitro (WST-1 assay, flow cytometry, or mammosphere-forming efficiency) results were analyzed using Student's $t$ test, and the Mann-Whitney $U$ test for unpaired samples. In vivo data were statistically evaluated by means of two-way analysis of variance (ANOVA). Significant treatment effects were subsequently delineated by using Dunnett's post hoc test. Statistical significance was assumed for $p<0.05$.

\section{Results \\ Cell viability of triple-negative cell lines treated with Cetuximab and Ixabepilone}

Cell viability was analyzed using the WST-1 assays of Cetuximab or Ixabepilone in the TNBC cell lines MDAMB-231 and SUM159 (Fig. 1). The viability of these cells did not change with increasing doses of Cetuximab (Fig. 1a and b). On the other hand, both cell lines were sensitive to Ixabepilone in a statistically significant dose-dependent manner $(p<0.01)$ (Fig. 1c and d). The inhibitory concentration (IC) $)_{50}$ was $10 \pm 3 \mathrm{nM}$ for MDA-MB-231 cells and $8 \pm 2$ nM for SUM159 cells. We found that Ixabepilone was the main contributor 

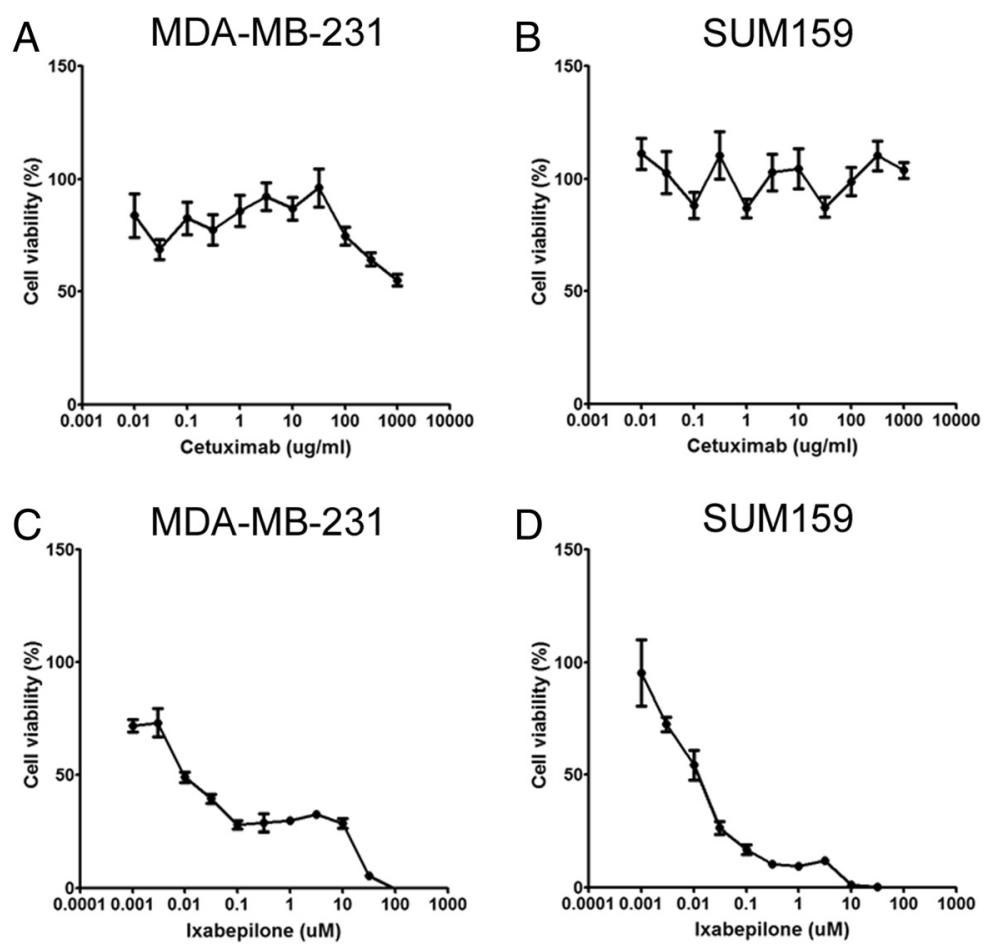

Fig. 1 The effect of Ixabepilone or Cetuximab on cell viability of triple-negative breast cancer cell lines with WST-1. WST-1 proliferation assay of MDA-MB-231 cells and SUM159 cells treated with Cetuximab (MDA-MB-231 cells (a) SUM159 cells (b)), or Ixabepilone (MDA-MB-231 cells (c), SUM159 cells $(\mathbf{d})$ ). The data for each cell line are mean \pm standard deviation obtained from three independent experiments

to cytotoxicity in the combination regimen of Cetuximab and Ixabepilone (data not shown).

\section{Breast CSCs are sensitive to Cetuximab}

We evaluated the effects of Cetuximab on the CSC population by analyzing $\mathrm{CD} 44^{+} / \mathrm{CD} 24^{-/ \text {low }}$, or $\mathrm{ALDF}^{+}$ populations using FACS. The tested doses of Cetuximab were 10,30 , and $50 \mu \mathrm{g} / \mathrm{ml}$. After treatment with Cetuximab for 3 days, a dose-dependent reduction in the $\mathrm{CD} 44^{+} / \mathrm{CD} 24^{-/ \text {low }}$ population of MDA-MB-231 cells was observed and the reduction was highest at a dose of $50 \mu \mathrm{g} / \mathrm{ml}$, from $89.8 \%$ to $35.8 \%(p<0.01)$ (Fig. 2a). Cetuximab also reduced the $\mathrm{ALDF}^{+}$population dose-dependently, with a maximum decrease from 2.2 to $0.4 \%(p<0.01)$ at $50 \mu \mathrm{g} / \mathrm{ml}$ of Cetuximab (Fig. 2b). Similarly, the highest concentration of Cetuximab reduced the ALDF+ population from 2.5 to $0.7 \%(p<0.01)$ in SUM159 cells in a dose-dependent manner (Fig. 2c).

\section{Cetuximab inhibits clonogenicity of TNBC cells}

Mammosphere-forming assays were performed to evaluate the effects of Cetuximab alone or in combination with Ixabepilone on MSFE of TNBC cells by measuring MSFE. The results showed that Cetuximab alone decreased MSFE in MDA-MB-231 cells from 0.24 to $0.13 \%$ $(p<0.05)$ and in SUM159 cells from $0.28 \%$ to $0.16 \%$, $(p<0.05)$ (Fig. 3a and b). Ixabepilone monotherapy had no effect on MSFE in either cell line. We also found that the combination therapy significantly reduced the MSFE of MDA-MB-231 cells at the $50 \mu \mathrm{g} / \mathrm{ml}$ dose of Cetuximab (the mean MSFE at Ixabepilone $5 \mathrm{nM}$ of $0.21 \%$ versus Ixabepilone $5 \mathrm{nM}+$ Cetuximab $50 \mu \mathrm{g} / \mathrm{ml}$ of $0.10 \%$, $p<0.05)$ (Fig. 3a). The combination treatment resulted in a reduction in MSFE for SUM159 cells, but the change was not statistically significant (mean at Ixabepilone $5 \mathrm{nM}$ of $0.30 \%$ versus Ixabepilone $5 \mathrm{nM}+$ Cetuximab $50 \mu \mathrm{g} / \mathrm{ml}$ of $0.20 \%, p>0.05$ ) (Fig. 3b). These results suggest that the EGFR signaling pathway may be critical for clonal expansion of CSCs.

\section{Tumor growth of TNBC xenografts with Cetuximab treatment}

We established human breast cancer cell xenografts in immunocompromised SCID/Beige mice using MDAMB-231 or SUM159, and divided them into four groups (control, Cetuximab, Ixabepilone, and combination). As shown in Fig. 4, Cetuximab therapy alone was effective in both MDA-MB-231 (tumor doubling time, TDT $=14 \pm 3$ days, $p<0.05$ ) and SUM159 (TDT $=30 \pm 10$ days, $p<0.05$ ) xenografts compared to control (TDT $=16 \pm 3$ days). In addition, the combined therapy induced a dramatic reduction in the tumor growth of SUM159 xenografts 


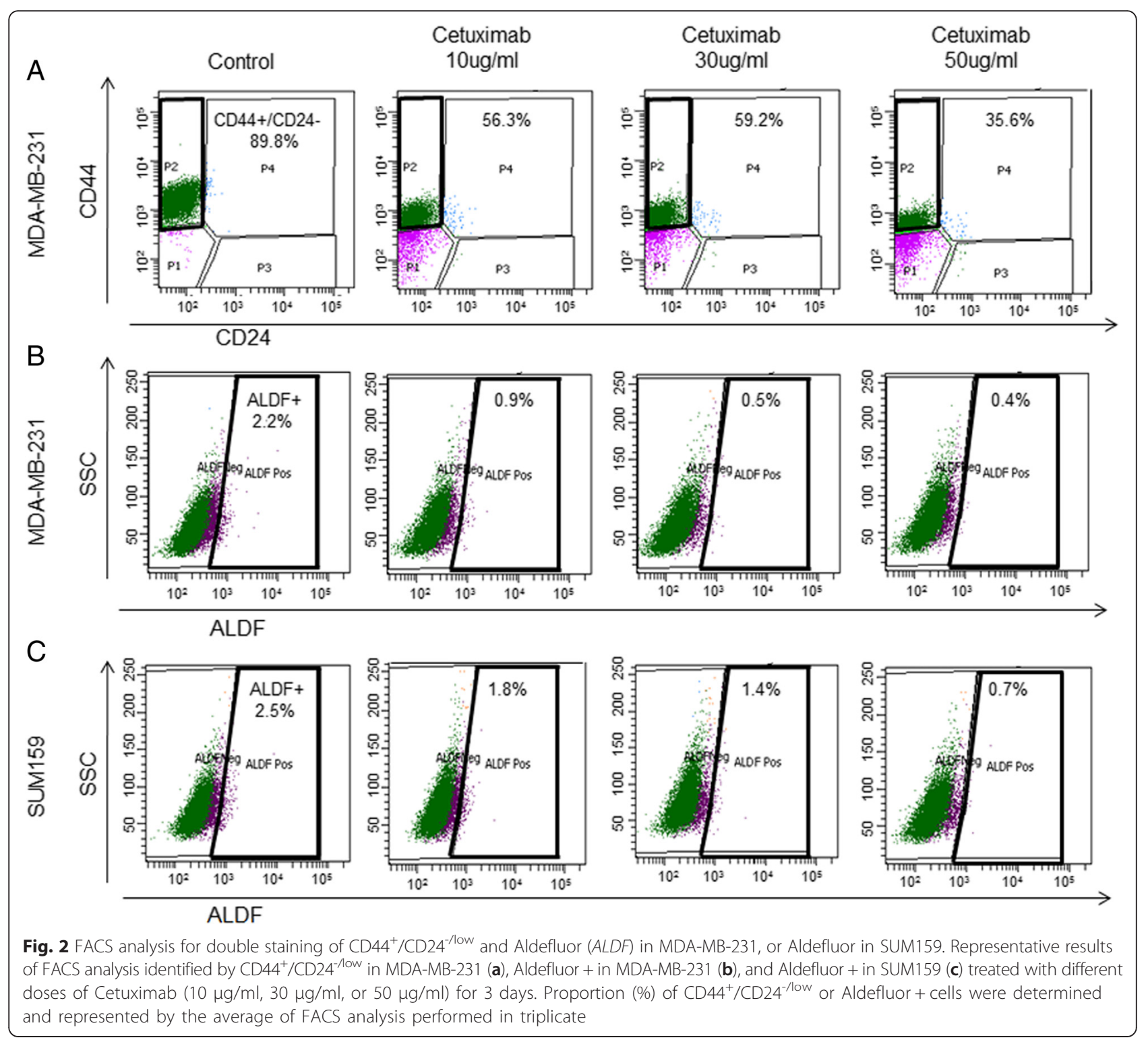

(TDT $=110 \pm 25$ days, $p<0.001)$ compared to Ixabepilone alone (TDT $=25 \pm 8$ days) (Fig. $4 \mathrm{~b}$ ). The combination treatment in MDA-MB-231 xenografts caused a decrease in tumor growth, but was not statistically significant (Fig. 4a). Also, the combination treatment was not toxic, and the weight curves of the combined treatment groups were similar to other groups in the xenografts (data not shown). These results suggest that Cetuximab is effective against CSCs, and treating TNBC xenografts with the combination therapy did not result in additive toxicity.

\section{Effects of Cetuximab treatment on breast CSCs in TNBC xenografts}

To evaluate the effect of Cetuximab, Ixabepilone, and combined treatment on breast CSCs in MDA-MB-231 and SUM159 xenografts, we performed both FACS analysis and MSFE assays of xenograft tumor specimens taken after treatment. In MDA-MB-231 xenograft tumors, the mean percentage of $\mathrm{CD} 44^{+} / \mathrm{CD} 24^{-/ \text {low }}$ cells decreased after treatment with Cetuximab $(31.8 \%, p<0.05)$ or the combination treatment $(29.3 \%, p<0.05)$ compared to control (56.1\%) (Fig. 5a). Of note, ALDF + cells were not identified in most of the MDA-MB-231 xenograft tumors (Fig. 5e). The MSFE of MDA-MB231 xenografts decreased after Cetuximab treatment $($ mean $=2.9 \%, p<0.01)$ or combination (mean $=0.8 \%, p<0.05$ ) compared to control (mean $=4.9 \%$ ) (Fig. 5c). On the other hand, the mean ALDF + percentage decreased by the combination treatment in SUM159 xenograft tumors (5.3 \%, $p=0.05)$ compared to control (12.4 \%) (Fig. 5b). However, $\mathrm{CD} 44^{+} / \mathrm{CD} 24^{-/ \text {low }}$ cells were not identified in most 


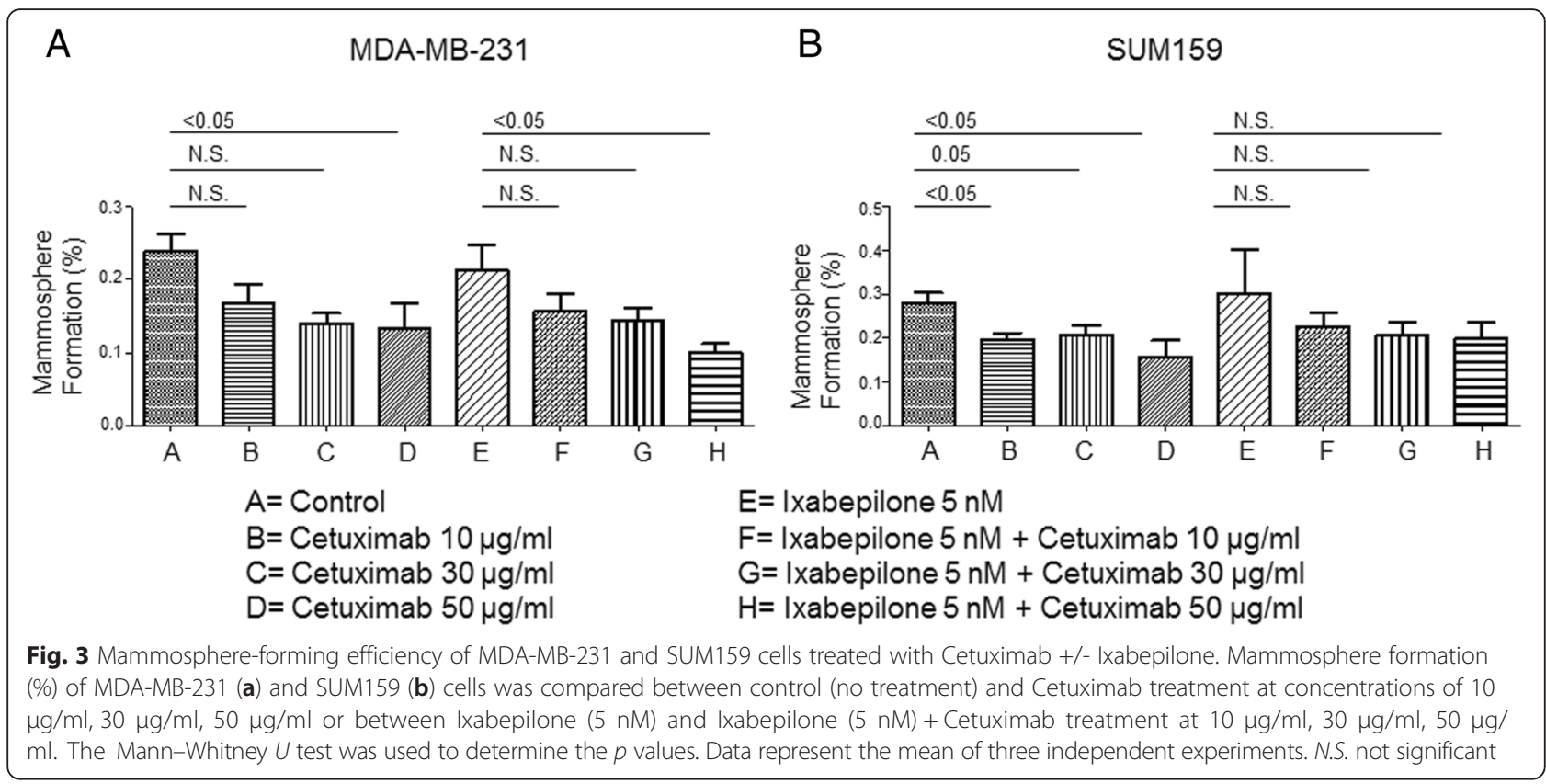

SUM159 xenograft tumors. Cetuximab treatment decreased the MSFE of SUM159 xenografts, but not to statistical significance (Fig. 5d). These results show that Cetuximab has the ability to decrease CSC populations in TNBC xenograft tumors.

\section{Cetuximab-treated tumors have decreased autophagy (LC3b, p62 and autophagosomes)}

To determine whether Cetuximab targets the EGFR signaling pathway and autophagy, we performed immunoblotting to determine the expression levels of EGFR, phospho-EGFR, LC3b-I and II, and p62 against the effects of Cetuximab, Ixabepilone, and the combination treatment in MDA-MB-231 and SUM159 cells (Additional file 1: Figure S1). Western blot analysis revealed that Ixabepilone treatment increased the expression of several proteins including EGFR, phospho-EGFR, phospho-AKT(473), and phospho-ERK1/2 in both cell lines. Cetuximab reduced phospho-AKT(473) and phospho-ERK1/2, which are downstream of EGFR in the signaling pathway. In addition, Cetuximab and especially the combination treatment downregulated the autophagy markers p62 and LC3b-II in MDA-MB-231 and SUM159 cells compared to control or Ixabepilone. Although Ixabepilone increased p62 expression, Ixabepilone either reduced or had no effect on the basal

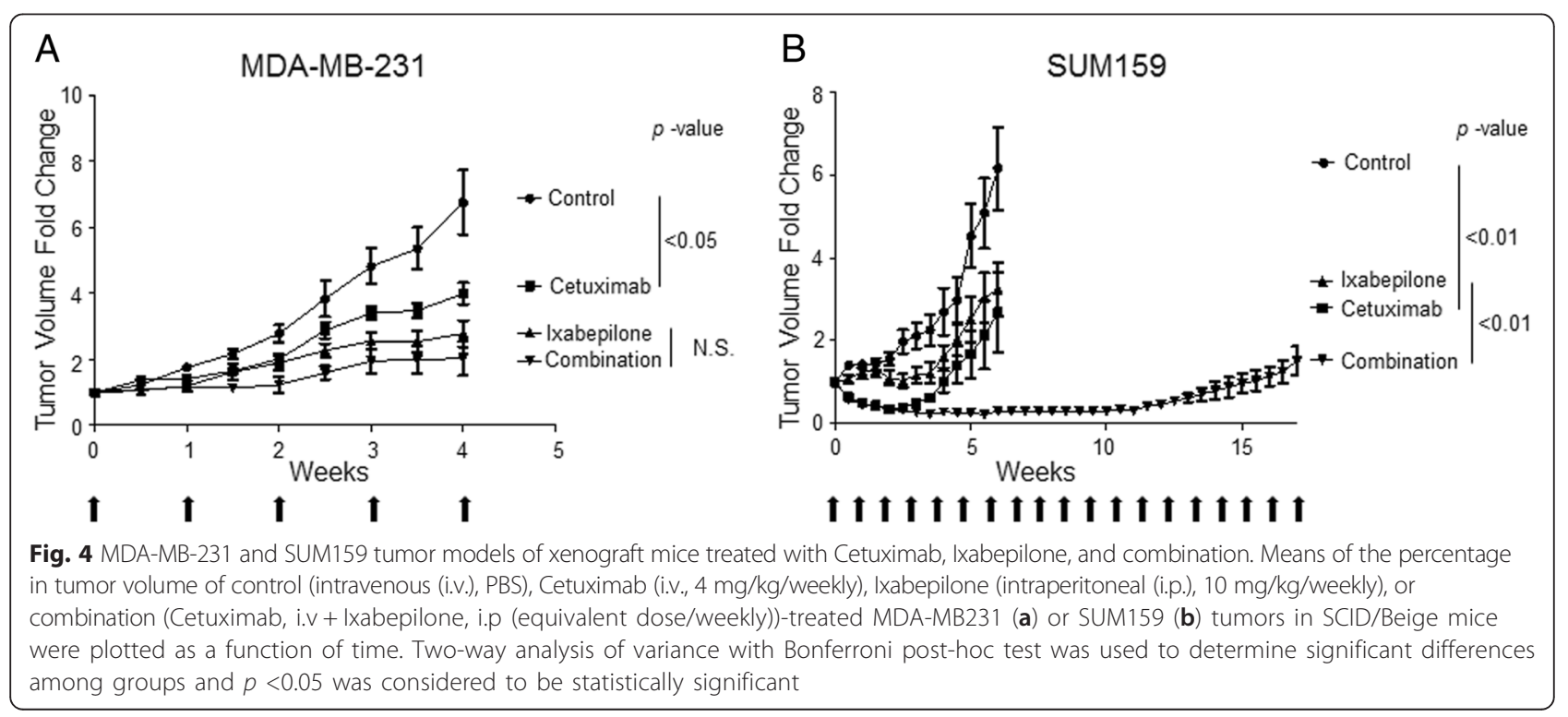




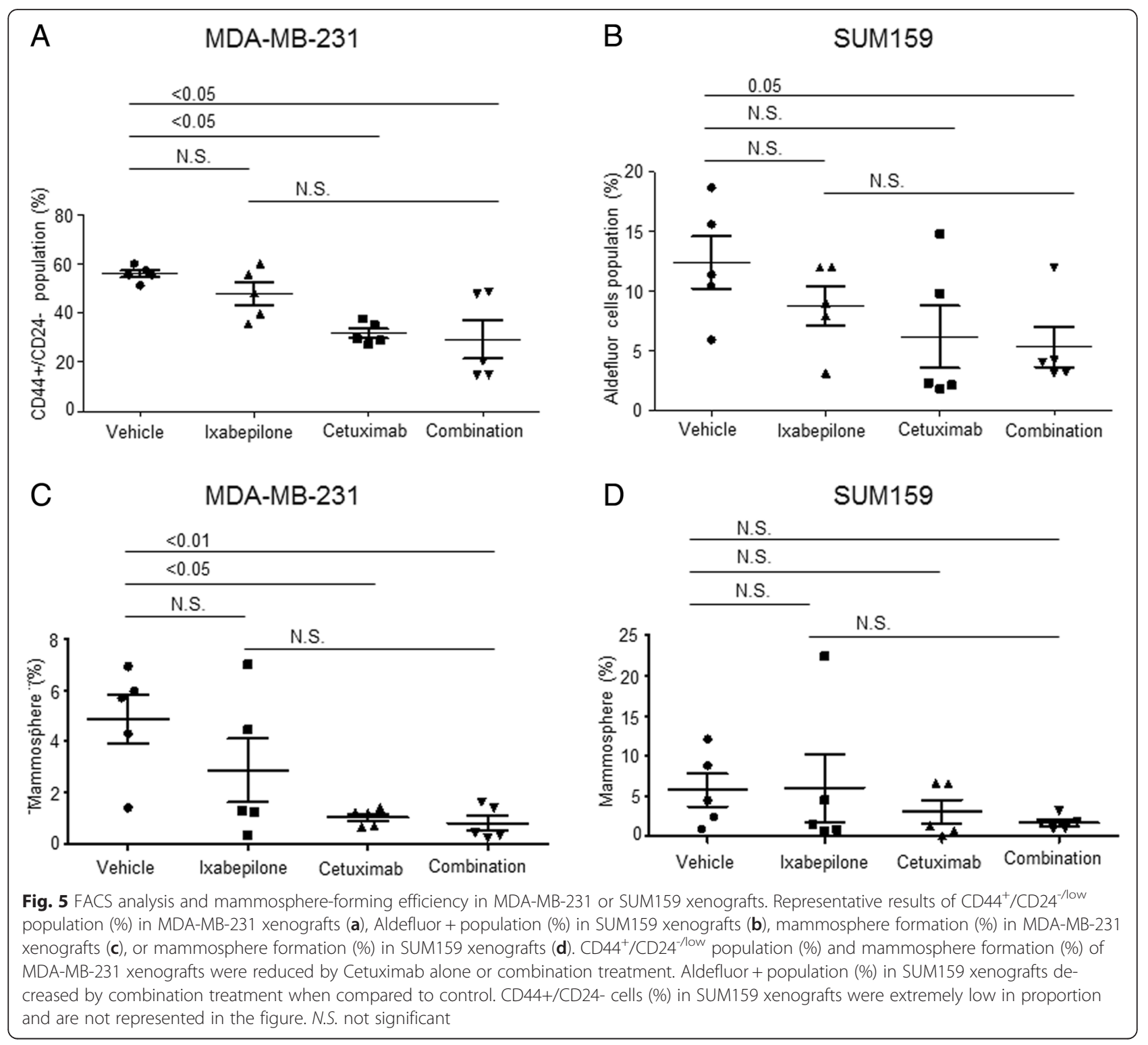

levels of LC3b-II in MDA-MB-231 and SUM159 cells, respectively. These results suggest that Ixabepilone upregulated autophagy. Together, these findings provide strong evidence that Cetuximab inhibits the Akt and Erk $1 / 2$ pathways, resulting in downregulation of p62 and LC3b. Moreover, the combination treatment decreased autophagy by inhibiting Ixabeplioneinduced EFGR activation.

\section{Discussion}

Tumorigenic CSCs are intrinsically resistant to conventional chemotherapy and have unique properties including enhanced self-renewal and increased propensity for tumor formation $[10,19]$. CSCs have been identified in various tumors including those of the breast, and they are particularly enriched in the basal-like and claudin-low subtypes of breast cancer. Cell lines representing the basal-like subtype (SUM159) and claudin-low subtype (MDA-MB-231) of breast cancer were utilized to study the anti-CSC effects of Cetuximab in combination with Ixabepilone. Our results indicate that Cetuximab alone or in combination with Ixabepilone significantly inhibited tumor growth and reduced $\mathrm{CD} 44^{+} / \mathrm{CD} 24^{-/ \text {low }} \mathrm{CSC}$ population in vitro and in vivo. However, the efficacy of the combination therapy varied in the two in vivo models compared to Ixabepilone monotherapy in that the effects of the combination were far greater in SUM159 tumors than in MDA-MB-231 tumors. Trédan et al. [20] recently reported that the clinical effectiveness of the Cetuximab and Ixabepilone combination was similar to that of Ixabepilone monotherapy in a first-line treatment of locally advanced or metastatic 
TNBC. Similarly, the results of two additional independent randomized phase II clinical trials, evaluating the effects of Cetuximab in combination with either cisplatin [21] or carboplatin [22], were similar to the clinical trial with Cetuximab and Ixabepilone. Carley et al. [22] separated patients into two groups, non-responders and responders, to the combination therapy by analyzing signaling signatures in post-treatment specimens. This revealed that low KRAS-amplicon expression was associated with a better response to the combination therapy of Cetuximab and carboplatin. In agreement with the results of the previous clinical trials, our data using two animal models reflect the disputed sensitivity of TNBC to Cetuximab in combination with chemotherapy. According to the Pietenpol TNBC classification [23], both cell lines are classified as mesenchymal-like. However, SUM159 cells have an H-Ras mutation and exhibit anaplastic carcinoma histology while MDA-MB-231 cells have a K-Ras mutation and invasive ductal carcinoma histology. These features may have contributed to the minimal response observed in MDA-MB-231 tumor models with the combination treatment. Additionally, differences in sensitivity to Ixabepilone may be another possible contributor to the bifurcated drug response with combination therapy, as our data indicate that SUM159 cells are more sensitive to Ixabepilone than MDA-MB-231 cells both in vitro and in vivo.

It was intriguing to observe that Cetuximab was not cytotoxic, but reduced CSCs in both cell lines in vitro and in vivo. This result is in agreement with previous reports that Cetuximab has little impact on cell viability $[24,25]$. We speculate that the EGFR signaling pathway may be critical to maintain the stemness of CSCs, and that loss of EGFR signaling may induce differentiation of the CSCs to a non-CSC population. This notion is supported by data from Harrison et al. [26], Yang et al. [27], and Gillian et al. [28], showing that EGFR signaling is critical for CSC stemness in human and murine breast CSCs. Previously, we reported that the treatment of patients with HER2-positive tumors using lapatinib (an EGFR/HER2 inhibitor) led to a statistically non-significant decrease in the percentage of $\mathrm{CD} 44^{+} / \mathrm{CD} 24^{-/ \text {low }}$ cells, and also a significant decrease in MSFE [29]. Despite effective targeting of the CSC population, our data suggested that inhibiting EGFR by Cetuximab in combination with Ixabepilone did not have significant anti-cancer effects in TNBC tumors. We hypothesize that either tumor heterogeneity or the development of compensatory mechanisms may have played a role in the minimal response to the Cetuximab and Ixabepilone combination. Supporting this hypothesis, Jacobsen et al. [30] recently showed that targeting EGFR can trigger compensatory activation of other ErbB family receptors, HER2 and/or HER3, and that simultaneous inhibition of EGFR,
ErbB2 and ErbB3 effectively overcame tumor heterogeneity and plasticity.

Previously, we reported that the CSC population increases after chemotherapy in both clinical and preclinical settings [17, 29, 31], and that autophagy is the underlying mechanism for the survival and maintenance of the CSC population [17]. In this report, we found that Ixabepilone treatment increased autophagy with a concomitant increase in EGFR signaling, while Cetuximab inhibited autophagy by reducing the expression of the autophagy markers p62 and LC3b. Therefore, we speculate that the inhibition of autophagy by Cetuximab may have played a role in reducing the CSC population. However, $\mathrm{Li}$ et al. have also shown that Cetuximab induces autophagy in A431 human vulvar squamous carcinoma cells, DiFi colorectal adenocarcinoma cancer cells, and $\mathrm{HCC} 827$ human non-small cell lung cancer cells by downregulating HIF- $1 \alpha$ and Bcl-2 and activating the beclin-1/hVps34 complex [32]. Thus, the regulation of autophagy by Cetuximab may differ depending on the type of cancer being studied.

\section{Conclusions}

These studies show that EGFR-targeted therapy effectively reduces CSC populations in SUM159 and MDAMB-231 tumors and tumor growth in vivo. However, our in vivo data suggest that the effects of the combination therapy of Cetuximab and Ixabepilone may vary based on the TNBC subtype and support recent clinical trial results. Thus, EGFR-targeted therapy should be considered with caution for TNBC patients, and further investigation of TNBC subtypes, using patient-derived xenograft TNBC models [33, 34], may help to determine TNBC patient groups suitable for the combination therapy.

\section{Additional file}

Additional file 1: Figure S1. Cetuximab treatment reduces autophagy. Western blot showed effects of cetuximab, ixabepilone, and combination (cetuximab + ixabepilone) treatment in MDA-MB-231 and SUM159 cells. Immunoblots were probed with antibodies against pEGFR, total EGFR, pARK, total ARK, p62, LC3b, pERK1/2, total ERK1/2, and b-Actin. Protein expression has been quantitated and normalized against the loading control, and the results of median of three replicates are represented in the table. (TIF $7 \mathrm{MB}$ )

\section{Abbreviations}

ALDF: Aldefluor; ALDH: aldehyde dehydrogenase; ANOVA: analysis of variance; CSC: cancer stem cell; DCIS: ductal carcinoma in situ; DMEM: Dulbecco's modified Eagle's medium; EGFR: epidermal growth factor receptor; ER: estrogen receptor; ERK: extracellular signal-regulated kinase; FACS: fluorescenceactivated cell sorting; HBSS: Hank's balanced salt solution; HER2: human epidermal growth factor receptor 2; i.p.: intraperitoneal; i.v.: intravenous; MBC: metastatic breast cancer; MEGM: mammary epithelial growth medium; MSFE: mammosphere formation efficiency; PBS: phosphate-buffered saline; PR: progesterone receptor; TDT: tumor doubling time; TNBC: triple-negative breast cancer. 


\section{Competing interests}

The authors declare that they have no competing interests.

\section{Authors' contributions}

Conception and design: TT, JCC. Development of methodology: T, DSC, JCC Acquisition of data: TT, DSC, AAR, LD. Data analysis and interpretation: TT, DSC, MDL, JCC. Writing, review, and/or revision of the manuscript: $T$, DHL, MG, DSC, JCC. All authors have read and approved the final manuscript.

\section{Acknowledgements}

We thank Dr. Gary Deyter for critical reading and editing of the manuscript. This research is supported by grants from the Breast Cancer Research Foundation; Komen Promise for the Cure KG081694; National Institutes of Health NIH U54 CA 149196; NIHR01 CA138197; Golfer's Against Cancer; Team Tiara; Causes for a Cure.

\section{Author details}

Methodist Cancer Center, Houston Methodist Hospital, 6445 Main Street, P21-34, Houston, TX 77030, USA. ²Department of Surgery, Houston Methodist Hospital, Houston, TX 77030, USA

\section{Received: 9 October 2014 Accepted: 14 December 2015}

\section{Published online: 12 January 2016}

\section{References}

1. Foulkes WD, Smith IE, Reis-Filho JS. Triple-negative breast cancer. N Engl J Med. 2010;363(20):1938-48

2. Hudis CA, Gianni L. Triple-negative breast cancer: an unmet medical need. Oncologist. 2011;16 Suppl 1:1-11.

3. Honeth G, Bendahl PO, Ringner M, Saal LH, Gruvberger-Saal SK, Lovgren K et al. The CD44+/CD24- phenotype is enriched in basal-like breast tumors. Breast Cancer Res. 2008;10(3):R53.

4. Frank NY, Schatton T, Frank MH. The therapeutic promise of the cancer stem cell concept. J Clin Invest. 2010;120(1):41-50.

5. Ward RJ, Dirks PB. Cancer stem cells: at the headwaters of tumor development. Annu Rev Pathol. 2007;2:175-89.

6. Reya T, Morrison SJ, Clarke MF, Weissman IL. Stem cells, cancer, and cancer stem cells. Nature. 2001;414(6859):105-11.

7. Lapidot T, Sirard C, Vormoor J, Murdoch B, Hoang T, Caceres-Cortes J, et al. A cell initiating human acute myeloid leukaemia after transplantation into SCID mice. Nature. 1994;367(6464):645-8.

8. Ginestier C, Hur MH, Charafe-Jauffret E, Monville F, Dutcher J, Brown M, et al. ALDH1 is a marker of normal and malignant human mammary stem cells and a predictor of poor clinical outcome. Cell Stem Cell. 2007;1(5):555-67.

9. Liu S, Cong Y, Wang D, Sun Y, Deng L, Liu Y, et al. Breast cancer stem cells transition between epithelial and mesenchymal states reflective of their normal counterparts. Stem Cell Reports. 2014;2:78-91.

10. Tanei T, Morimoto K, Shimazu K, Kim SJ, Tanji Y, Taguchi T, et al. Association of breast cancer stem cells identified by aldehyde dehydrogenase 1 expression with resistance to sequential Paclitaxel and epirubicin-based chemotherapy for breast cancers. Clin Cancer Res. 2009;15(12):4234-41.

11. Castanieto A, Johnston MJ, Nystul TG. EGFR signaling promotes self-renewal through the establishment of cell polarity in Drosophila follicle stem cells. eLife. 2014;3.

12. Roche H, Vahdat LT. Treatment of metastatic breast cancer: second line and beyond. Ann Oncol. 2011;22(5):1000-10.

13. Rak Tkaczuk KH. Ixabepilone as monotherapy or in combination with capecitabine for the treatment of advanced breast cancer. Breast Cancer. 2011:5:1-14

14. Sparano JA, Vrdoljak E, Rixe O, Xu B, Manikhas A, Medina C, et al. Randomized phase III trial of ixabepilone plus capecitabine versus capecitabine in patients with metastatic breast cancer previously treated with an anthracycline and a taxane. J Clin Oncol. 2010;28(20):3256-63.

15. Dumontet $C$, Jordan MA, Lee FF. Ixabepilone: targeting betalll-tubulin expression in taxane-resistant malignancies. Mol Cancer Ther. 2009:8(1):17-25.

16. Vahdat L. Ixabepilone: a novel antineoplastic agent with low susceptibility to multiple tumor resistance mechanisms. Oncologist. 2008;13(3):214-21.

17. Choi DS, Blanco E, Kim YS, Rodriguez AA, Zhao H, Huang TH, et al. Chloroquine eliminates cancer stem cells through deregulation of Jak2 and DNMT1. Stem Cells. 2014;32(9):2309-23.
18. Granados-Principal S, Liu Y, Guevara ML, Blanco E, Choi DS, Qian W, et al. Inhibition of iNOS as a novel effective targeted therapy against triplenegative breast cancer. Breast Cancer Res. 2015;17:25

19. Dean M, Fojo T, Bates S. Tumour stem cells and drug resistance. Nat Rev Cancer. 2005;5(4):275-84

20. Tredan O, Campone M, Jassem J, Vyzula R, Coudert B, Pacilio C, et al. Ixabepilone alone or with cetuximab as first-line treatment for advanced/ metastatic triple-negative breast cancer. Clin Breast Cancer. 2015;15(1):8-15.

21. Baselga J, Gomez P, Greil R, Braga S, Climent MA, Wardley AM, et al. Randomized phase II study of the anti-epidermal growth factor receptor monoclonal antibody cetuximab with cisplatin versus cisplatin alone in patients with metastatic triple-negative breast cancer. J Clin Oncol. 2013;31(20):2586-92.

22. Carey LA, Rugo HS, Marcom PK, Mayer EL, Esteva FJ, Ma CX, et al. TBCRC 001: randomized phase II study of cetuximab in combination with carboplatin in stage IV triple-negative breast cancer. J Clin Oncol. 2012;30(21):2615-23.

23. Lehmann BD, Bauer JA, Chen X, Sanders ME, Chakravarthy AB, Shyr Y, et al. Identification of human triple-negative breast cancer subtypes and preclinical models for selection of targeted therapies. J Clin Invest. 2011;121(7):2750-67.

24. Corkery B, Crown J, Clynes M, O'Donovan N. Epidermal growth factor receptor as a potential therapeutic target in triple-negative breast cancer. Ann Oncol. 2009:20(5):862-7.

25. Brand TM, lida M, Dunn EF, Luthar N, Kostopoulos KT, Corrigan KL, et al. Nuclear epidermal growth factor receptor is a functional molecular target in triple-negative breast cancer. Mol Cancer Ther. 2014;13(5):1356-68.

26. Harrison H, Simoes BM, Rogerson L, Howell SJ, Landberg G, Clarke RB. Oestrogen increases the activity of oestrogen receptor negative breast cancer stem cells through paracrine EGFR and Notch signalling. Breast Cancer Res. 2013:15(2):R21.

27. Yang J, Liao D, Chen C, Liu Y, Chuang TH, Xiang R, et al. Tumor-associated macrophages regulate murine breast cancer stem cells through a novel paracrine EGFR/Stat3/Sox-2 signaling pathway. Stem Cells. 2013;31(2):248-58.

28. Farnie G, Clarke RB, Spence K, Pinnock N, Brennan K, Anderson NG, et al. Novel cell culture technique for primary ductal carcinoma in situ: role of Notch and epidermal growth factor receptor signaling pathways. J Nat Cancer Inst. 2007;99(8):616-27.

29. Li X, Lewis MT, Huang J, Gutierrez C, Osborne CK, Wu MF, et al. Intrinsic resistance of tumorigenic breast cancer cells to chemotherapy. J Natl Cancer Inst. 2008;100(9):672-9.

30. Jacobsen HJ, Poulsen T, Dahlman A, Kjaer I, Koefoed K, Sen JW, et al. Pan-HER, an Antibody Mixture Simultaneously Targeting EGFR, HER2, and HER3, Effectively Overcomes Tumor Heterogeneity and Plasticity. Clin Cancer Res. 2015;21(18):4110-22.

31. Creighton CJ, Li X, Landis M, Dixon JM, Neumeister VM, Sjolund A, et al. Residual breast cancers after conventional therapy display mesenchymal as well as tumor-initiating features. Proc Natl Acad Sci USA. 2009;106(33):13820-5.

32. Li X, Fan Z. The epidermal growth factor receptor antibody cetuximab induces autophagy in cancer cells by downregulating HIF-1alpha and $\mathrm{BCl}-2$ and activating the beclin 1/hVps34 complex. Cancer Res. 2010;70(14):5942-52.

33. Zhang X, Claerhout S, Prat A, Dobrolecki LE, Petrovic I, Lai Q, et al. A renewable tissue resource of phenotypically stable, biologically and ethnically diverse, patient-derived human breast cancer xenograft models. Cancer Res. 2013;73(15):4885-97.

34. Landis MD, Lehmann BD, Pietenpol JA, Chang JC. Patient-derived breast tumor xenografts facilitating personalized cancer therapy. Breast Cancer Res. 2013;15(1):201. 